\title{
Electronic Forms-Based Computing for Evidentiary Analysis
}

Andy Luse

lowa State University

Brian Mennecke

lowa State University

Anthony M. Townsend

lowa State University

Follow this and additional works at: https://commons.erau.edu/jdfs|

Part of the Computer Engineering Commons, Computer Law Commons, Electrical and Computer Engineering Commons, Forensic Science and Technology Commons, and the Information Security Commons

\section{Recommended Citation}

Luse, Andy; Mennecke, Brian; and Townsend, Anthony M. (2009) "Electronic Forms-Based Computing for Evidentiary Analysis," Journal of Digital Forensics, Security and Law. Vol. 4 : No. 3 , Article 2.

DOI: https://doi.org/10.15394/jdfsl.2009.1061

Available at: https://commons.erau.edu/jdfsl/vol4/iss3/2

This Article is brought to you for free and open access by

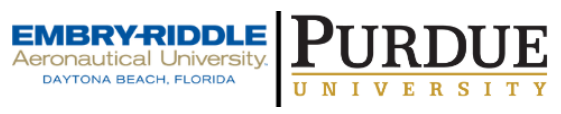
the Journals at Scholarly Commons. It has been accepted for inclusion in Journal of Digital Forensics, Security and Law by an authorized administrator of Scholarly Commons. For more information, please contact commons@erau.edu.

(c)ADFSL 


\title{
Electronic Forms-Based Computing for Evidentiary Analysis
}

\author{
Andy Luse \\ Iowa State University \\ andyluse@iastate.edu \\ Brian Mennecke \\ Iowa State University \\ mennecke@iastate.edu \\ Anthony M. Townsend \\ Iowa State University \\ amt@iastate.edu
}

\begin{abstract}
The paperwork associated with evidentiary collection and analysis is a highly repetitive and time-consuming process which often involves duplication of work and can frequently result in documentary errors. Electronic entry of evidencerelated information can facilitate greater accuracy and less time spent on data entry. This manuscript describes a general framework for the implementation of an electronic tablet-based system for evidentiary processing. This framework is then utilized in the design and implementation of an electronic tablet-based evidentiary input prototype system developed for use by forensic laboratories which serves as a verification of the proposed framework. The manuscript concludes with a discussion of implications and recommendations for the implementation and use of tablet-based computing for evidence analysis.
\end{abstract}

Keywords: forms-based computing, tablet pc, evidence analysis

\section{INTRODUCTION}

Forensic laboratories' documentary processes are constantly under scrutiny and stakeholder expectations are always high. Analysts and managers in forensics laboratories face this challenge at the same time that they must contend with everincreasing demands for shorter cycle times for evidentiary analysis. Electronic recordkeeping mechanisms have helped to alleviate some of the pressure by providing databases and terminal-based entry of data pertaining to evidence management and evidentiary findings. While these systems provide outstanding support for data that is already in the system, the problem still remains that the primary method for both initial evidence gathering and subsequent analysis relies 
on paper for collecting and sometimes storing written records. This increases the likelihood of errors as data from paper forms is entered into LIMs and other lab systems, and adds to overall workload as the same data is re-entered at multiple points in the evidentiary collection and analysis process.

By obviating the data entry redundancies of paper-to-electronic workflows, electronic tablet-based input mechanisms provide a means for both quality improvement and faster turnaround time for evidentiary analysis, while maintaining the look, feel, and familiarity of traditional paper forms. Electronic forms offer a single point of data entry at, for example, the crime scene, which brings data entry right to the scene of investigation. In most information systems designed to capture data about transactional events, the goal is to automate data collection and move it as close in time and space to the point at which the transaction or event occurs, and to eliminate re-entry of data, which avoids problems of inefficiency and transcription error.

This paper is structured as follows. Background information is given pertaining to research in paperless offices, electronic laboratory notebook implementations utilized by other scientific disciplines, and examples of the successful use of electronic forms and electronic tablets in other fields. A framework for the implementation of an electronic tablet-based evidentiary collection and analysis system for forensic laboratories is then proposed. Prototype development of an electronic tablet-based evidentiary collection and analysis system is described. A discussion follows which provides contributions of the research as well as recommendations for those interested in developing such systems. Finally, concluding comments are given.

\section{BACKGROUND}

This section provides an overview of background literature pertinent to this study. Various areas are also detailed where electronic forms have been utilized to allow for improvements in data entry.

\subsection{Paperless Office}

The idea of the paperless office has been touted for years as the ultimate goal of any successful records management initiative, with numerous articles describing methods for implementing paperless offices. One of the oft cited articles on paperless offices is Hough's (1989) description of a system for storing, retrieving, and manipulating document images. Lau (1990) outlines a method for building a paperless office using document imaging. Conferences have even held exercises designed to develop methods for converting to a paperless system (Patterson and Olah 1981). A few success stories can be found where part or all of a paperless office system lived up to expectations (Hunton 1994; Phelan 2003). The problem 
still remains that these success stories are few and far between and the successful prototype of the paperless office is still a dream, particularly in the forensic community.

Many other examples show that the paperless office has failed to live up to the expectations of many employees, managers, and corporations. In (Sellen and Harper 2002), the authors provide an in-depth analysis documenting reasons for the failure of the paperless office. This is explained through the affordances of paper, or the fundamental recording and manipulation activities that paper allows or affords. The physical properties of paper (lightweight, thin, flexible, etc.) allow it to be used in a number of ways including grasping, carrying, folding, writing, etc. While computing technologies have allowed for the mass proliferation of information, the physical properties of a computer make it unwieldy for certain traditionally paper-based tasks. Therefore, many still incorporate paper for reading, note taking, and many other activities for which the tactile and flexible characteristics of paper are more suitable.

Forensic laboratory environments are a prime candidate for paperless deployment. Because most data pertaining to both evidence collection and analysis must eventually be entered into a backend database management system, paper-based records are an unnecessary step in the process. Warner (2007) provides a description showing how a laboratory environment can save time and money by retooling their business processes and other aspects of their corporate environment towards electronic records. We have applied these ideas associated with electronics forms-based computing to criminalist laboratories and documented the paperless process in Figure 1. The benefits of applying EFBC to crime laboratories are numerous, but chief among these is the fact that the data collection process is moved closer to the source of the data (i.e., the crime scene) and the data are automatically entered into the LIMS with little or no error. By moving data collection and entry to the primary source of the data, error rates will be reduced, time loss due to redundant data entry will be reduced, and money will be saved.

\subsection{Military Electronic Notebook Use}

The use of electronic notebooks by the military is an excellent indicator of the viability of electronic notebook systems in forensic practice. Like field investigators, military operations require a level of ruggedness and reliability far in excess of most civilian operations. Various military applications have employed the use of electronic notebooks for process improvement. Electronic notebooks are particularly useful for military applications which are typically highly mobile in nature. Electronic notebooks allow operators to perform their job without having to leave the field or lug cumbersome equipment along. 
Electronic notebooks have been extensively utilized for coordination of flightline operations (Faas, Seyba et al. 2006). This allows personnel in the field to coordinate with others to schedule the necessary resources - including personnel, test equipment, spare parts, etc. - for an upcoming flight. The Warrior's Edge Soldier System (WESS) is another system which has been developed to provide situational awareness for both soldiers and command (Walrath 2005). The system provides a method for blue force tracking (or tracking of friendly personnel) by allowing collaboration between both soldiers in the field and those at the base.

\subsection{Medical Electronic Forms Entry Systems}

The medical profession has seen a great deal of integration with technology in all operations surrounding patient care. Furthermore, the growth rate for IT in this area is phenomenal and will continue to grow at an increase rate for the foreseeable future (Raghupathi and Tan 2002). Mobile access to information, utilizing electronic forms mechanisms, is one of these growth areas. It has been shown that these mobile technologies offer the potential for improvements in information access, workflow enhancement, and better decision-making at the point of care (Lu, Xiao et al. 2005) while eliminating double documentation (Ammenwerth, Buchauer et al. 2000). Electronic forms have also been shown to improve accuracy, quality, and level of detail for patient records (Miller and Sim 2004; Wu and Straus 2006).

\subsection{Electronic Tablets in Education}

Since their inception, tablet PCs have been viewed as an educational tool, particularly in a university context, for both faculty and students alike. The technology offers a more familiar experience which parallels traditional handwritten notes, while integrating the user with information technology resources. Studies have looked at the strengths of tablet PCs in this environment for note-taking, presentations, document markup, and information management (Willis and Miertschin 2004). Students have found the use of electronic tablets useful in a classroom environment by allowing for more robust notes than a typical laptop computer, while also allowing for ease of searching for particular content, whether written with a stylus or typed (Frolik and Zurn 2005). Students have also shown very positive feedback towards the use of tablet PCs by faculty utilized in classroom environments (Wise, Toto et al. 2006).

\subsection{Electronic Laboratory Notebooks}

Electronic laboratory notebooks (ELNs) combine the flexibility of a paper notebook with the power of personal computing, database systems, and computer networks (Myers, Fox-Dobbs et al. 1996). By combining these features, the 
evidence analysis process can follow a smooth path from initial entry to subsequent analysis and use. Tablet computers are a central component of ELNs. Specifically, the tablet becomes a tool for not only analysis but also for data collection and presentation.

ELNs have been utilized in many disciplines including chemistry (Hughes, Mills et al. 2004; Schraefel, Hughes et al. 2004), biology (Arnstein, Hung et al. 2002; Mackay, Pothier et al. 2002), as well as general ELNs for a variety of scientific areas (Myers, Fox-Dobbs et al. 1996; Myers 2003). These ELNs provide a valuable asset for use in laboratory environments. The primary difference between these electronic tablets and those for use in a forensic laboratory is that the majority of these other ELNs were designed for mobile office or controlled climate conditions. While some of the ELNs described in the literature are able to be moved around, for example, a laboratory, they are not designed to be taken into the "field." In fact, the ELNs described in the literature do not make use of tablet PC technology, but rather, use conventional notebooks. Thus, the forensic electronic notebook technology we describe here is unique. Specifically, these tablets must be designed to be highly mobile in harsh weather conditions as well as capable of data synchronization between field locations and a centralized database housed in the laboratory. Finally, they must also afford the user the same kind of ease-of-use that has been the hallmark of traditional paper-based systems.

\section{PROPOSED FRAMEWORK}

This section will be used to describe a proposed forms-based electronic tablet system for use in a forensic laboratory environment. This framework is divided into five specific areas based on the major decision points which need to be made when implementing such a system (see Table 1). Each decision point will be described with possible implementation methods as well as pros and cons associated with the methods described. Some of these pieces can also be identified in the context of the overarching evidentiary process, with a comparison to evidentiary analysis without a forms-based electronic tablet system, in Figure 1 below.

Table 1. Decision points for implementing a forms-based electronic tablet system in a forensic laboratory.

\begin{tabular}{|l|l|}
\hline Decision & Explanation \\
\hline Data & What data needs to be entered at all stages of analysis \\
\hline Current system & Can the current system handle it? What needs to be modified? \\
\hline Forms & What forms are needed? Who needs them? How should they be laid out? \\
\hline Technology & What technology will be utilized? \\
\hline Development & Development of the entire solution. \\
\hline
\end{tabular}




\subsection{Data}

The first step in the framework for electronic forms-based implementation involves the identification of the data requirements. Most labs have many of the same basic data requirements as other labs, but there are always a few exceptions. Therefore, an analysis of the current data needs for the lab should be thoroughly examined. This will involve interviews with employees at all levels which input any type of data. Also, many labs have a current database system which information is entered into once the examination or analysis has been completed. This may be the most concrete representation of the data needed. Current needs may require the use of this system, or if a new system is being developed, this will serve as a good starting point.

\subsection{Current System}

The next step in the process is examining the actual system that is currently in use. Most labs will have some sort of backend LIMS system for keeping records. First, an assessment of this system will need to be done to evaluate whether it is capable of allowing integration with electronic tablets. Future needs, such as barcode or RFID evidence tracking should also be looked at to allow for easier integration of these items in the future. Access mechanisms will also need to be assessed to verify that the necessary infrastructure is available for wireless access from within the lab by electronic tablets. Security will also need to be assessed to verify that the necessary restrictions are in place to accommodate wireless entry of data.

\subsection{Forms}

The most important thing to remember when designing the electronic forms is to mimic, as closely as possible, extant paper-based forms. Users will be more comfortable with an electronic form that is familiar and this will cause less tension during the transition to the new system.

Next, remember to design all forms for the point in the process where they will be used. There are many different forms used for evidentiary collection and analysis. First, decide which fields are necessary. Second, combine multiple forms if space allows. Forms should be designed with multiple tabs to represent separate pages when more than one form is currently used.

Finally, decide on what type of form entry is most suitable for the backend data definitions decided upon in step 1. Are there requirements for the length or type of data entered (i.e. 2 alphabetic characters for a state)? Is there a way in which errors in data entry can be avoided (i.e. use of a calendar control to select a date and/or time)? Are there certain visual fields which may better reflect the data (i.e. a check box for a True/False field)? 


\subsection{Technology}

There could be a large variety of technology needed based on the amount of current technology being used and the size of the lab. The primary reason for choosing the technology at this step is to ensure that the technology fits what is needed by the system and not the other way around. Designing a good system must be the foremost priority as there are many different types of technologies which can be utilized for most any situation. The following is a list of possible items needed.

Server technology - If this is the first technology implemented for evidence analysis, or more realistically, a new backend system will be implemented along with the electronic tablet system, then a server will be needed. There are many different technologies which can be implemented based on current technical expertise and available funding. For example, servers based on a Microsoft or Linux architecture can be considered. Factors such as price, staff familiarity, system compatibility, and similar considerations should be evaluated in selecting a platform. Regardless of the platform chosen, a database system will need to be installed. Oracle provides a robust installation for large data needs. Microsoft SQL Server also offers an enterprise-level system. Free products such as Microsoft's SQL Server Express edition and mySQL are also viable alternatives. It is key that the chosen technology should meet the needs for this project and be scalable for the future.

Networking - The next step is supplying connections to the server/database. This should include both wire-based connections (Ethernet) as well as wireless connections for easy upload and use of information from the electronic tablets. Here, an important consideration is security. Due to the sensitive nature of the data handled by laboratory personnel, the connections to the server should be as secure as possible. This is even more important with wireless connections. A RADIUS server is recommended for authentication over the wireless network with encryption such as WPA.

Electronic tablets - After the backend infrastructure has been decided on, the tablet technology should be selected. Due to the distinctive nature of forensic examination, special tablets will be needed, especially in the field. As mentioned above, ruggedized tablets are needed to withstand the multitude of conditions which could be encountered in the field. Be sure to define specifications for a tablet that is designed to be able to withstand extreme weather conditions, including extreme heat and cold (e.g., most equipment will be stored in vehicles). For laboratory applications, it is possible that less expensive non-ruggedized tablets can be used; however, ruggedized tablets may also be needed in laboratory settings for situations where sterilization will be needed to prevent crosscontamination. It should also be stressed that tablet computers, which use penbased entry, are far superior for field use than conventional laptops. Tablet machines function for the user like a clipboard and form, allowing the user to be 
mobile and work standing up.

Peripherals - Select any peripherals which are needed that can be operated with the configured system. This may include such things as barcode scanners, cameras, etc.

\subsection{Development}

Finally, when all of the technology is decided upon, the system will need to be assembled. Each part above will need to be configured and setup. This is highly dependent on the technology and the software utilized. The main portions which are needed by the electronic tablet system will be the database and the formsbased programs.

The forms-based program is highly dependent on the above analyses. The system should mimic the forms and data entry process/restrictions decided upon during step 2. The key is to verify that any components and applications adhere to the requirements decided upon during the initial analysis phase of the project.

\section{FIELD \CASE STUDY}

Our team obtained information for this study from several sources during this project as well as during a previous MFRC funded project that focused on an examination of the features and capabilities of LIMS. During the last two years we have had the opportunity to make observations of laboratory operations, to have discussions with numerous laboratory stakeholders, and to engage in structured discussions with a variety of employees in these facilities. In total, our research group visited nine (9) Midwestern crime laboratories during the summer of 2005 to discuss LIMS and RFID issues (see Ames Laboratory Report No. IS5175 for further information). The researchers also visited two (2) crime laboratories (Johnson County, Missouri, and the Massachusetts State Police Forensics Laboratory) during the summer of 2006 to specifically discuss RFID. At each location, several of us met with the management team and with numerous scientific, technical, and administrative employees in semi-structured information gathering sessions. In addition, during the summer of 2005 researchers visited Porter Lee, Inc., the manufacturer of the Beast, to discuss LIMS and data management issues. Similarly, we also conducted a conference call with representatives of Forensics Technologies, Inc. (FTI), the makers of the BARD LIMS, to discuss LIMS and RFID. Although all of these site visits were keyed to the primary projects of LIMS or RFID investigation, the research team was also able during each of these visits to examine documentary flow and data entry systems, both as they related to the primary task, as well as to determine the viability of electronic forms.

This field-based portion of our research provided us with critical information 
about document generation and data entry in laboratories. First, it underscored the data management challenges that laboratories face in relation to field data collection, data in-take, data management, and data analysis. Second, it allowed us to examine and document the processes used to process and manage data and evidence as it flowed into, through, and out of the laboratory.

The above analyses provided information for the development of a prototype system for electronic forms-based data entry for evidentiary collection and analysis. The framework described earlier also provided a structured agenda for the development of the prototype. This case study, describing the implementation of a working prototype, serves as a model for utilization of the proposed framework.

The first step involved identification of the data requirement decisions necessary at each step in the collection and analysis process. Interviewees were questioned about the information they recorded at evidence collection, data entry points into the laboratory information system (i.e., the LIMS), and during subsequent analyses and data entry points. This data was then analyzed for thoroughness and compared with current paper-based forms utilized for evidence collection and analysis at all steps in the forensic process. Any new information deemed necessary by our interviewers was also analyzed. Based on these interviews and analyses, a database model was developed representing the data collected during the process and the various entities which comprised this data.

Once the appropriate data fields were decided upon, the next step focused on identifying the types (i.e., integer, string, Boolean, etc.) and values for these fields. If a field would only have a certain subset of values, these were recorded. Also, other special fields, such as true/false fields, were identified and classified. Each field was also designated a data type such as number, character string, etc. This is an important step in the development process, because it provides the basis of the electronic form's capacity to "self-correct" erroneous data entry as it occurs; unlike paper, the electronic form recognizes when an inappropriate entry is placed into a field, and requires the user to correct the entry.

The data was also separated into groups corresponding to where in the process the data was entered (a high-level overview of this process can be seen in Figure 1). This involved an analysis of the database model as well as current paper-based forms. These forms offered a snapshot of the data needed based on their current use in the progression of the forensic analysis. 


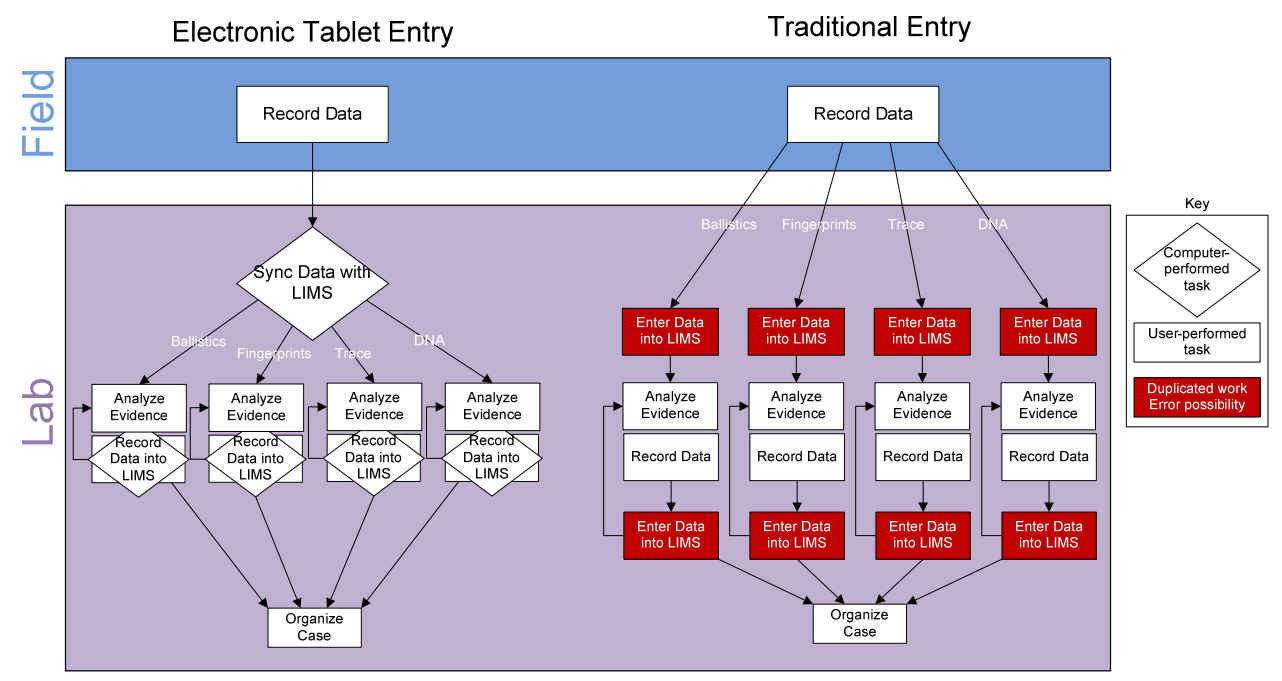

Figure 1 . The data entry process utilizing electronic tablet entry and analysis and traditional entry and analysis.

Third, we designed mockups of electronic versions of the various forms used for data entry/modification at each step in the evidentiary analysis process. Current paper-based forms were used as a starting template for the forms implemented on the electronic tablet. Also, data requirements at each step in the process were used as a template. The mode of entry was analyzed based on the type of the data entered as well as whether there were a finite number of possible entry values. For example, a dropdown list was decided upon for entry of the forensic personnel collecting and/or analyzing the evidence because each analyst must already be in the system before he or she can perform an analysis.

Fourth, the technology requirements were examined and identified. The new system needed to be as similar to paper-based recording as possible, yet still adhere to the features and capabilities inherent in paperless systems. Also, because the system was to be used in the field, it needed to be ruggedized to any possible outdoor elements. We decided upon the ruggedized Xplore laptop series (http://www.xploretech.com/index.pl?id=2273) due to its testing for rain, temperature, dust, and many other environmental factors which may be present in the field. Microsoft Windows XP Tablet Edition was utilized as the client operating system on the tablets. Also, a local Access database was used to temporarily store form data in the field before the analyst was able to come back to the lab and sync it with the primary data store. The server technology for the lab utilized a Windows Server 2003 architecture with SQL Server 2005 as the data repository. The tablets were able to utilize $802.11 \mathrm{~g}$ wireless technology to sync data with the server upon returning to the lab. Also, lab examiners were able to utilize this same wireless capability to allow for untethered electronic forms- 
based entry of information during analysis. ${ }^{1}$

Finally, the data model, the process, and the other requirements were implemented using the above technology. First, server and networking technologies were designed to allow access to the database server at the lab. Next, the data stores were implemented for the tablets while in the field (using Access) and for the primary storage server (using SQL Server). Finally, using .NET forms-based programming for tablet PC, we implemented the forms which we had designed above. Currently, the primary operating system for tablet computers is Microsoft, so their .NET programming language provides a wellintegrated programming solution. A sample of one such form can be seen in Figure 2.

${ }^{1}$ Other peripheral technologies such as the Zebra QL 420 belt printer for tagging items

(http://www.zebra.com/id/zebra/na/en/index/products/printers/mobile/q

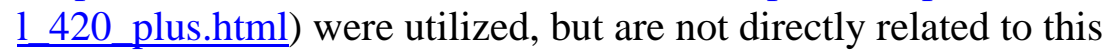
study. 
Journal of Digital Forensics, Security and Law, Vol. 4(3)

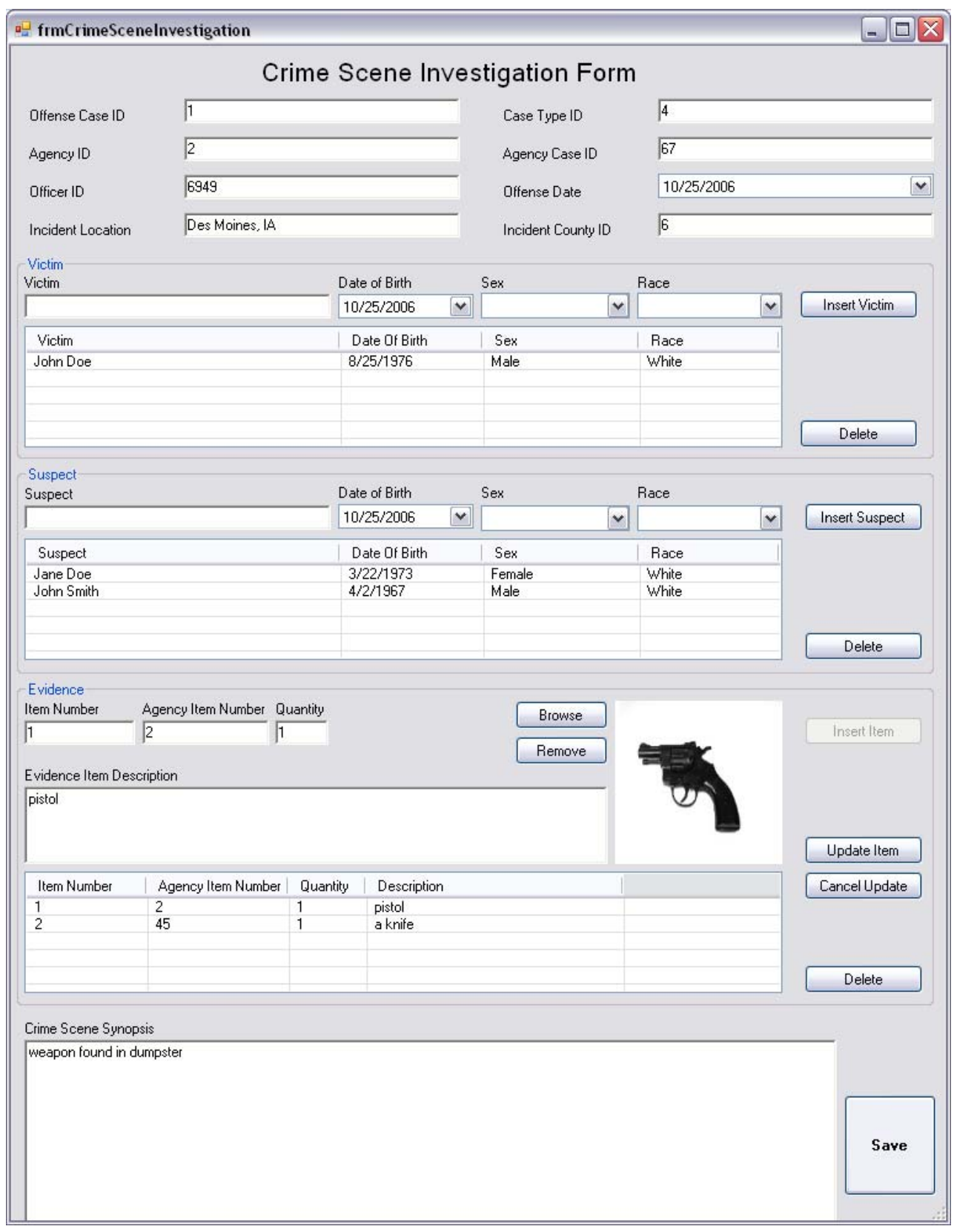

Figure 2. Example electronic form for evidentiary entry 


\section{DISCUSSION}

\subsection{Contributions}

The proposed electronic tablet-based evidentiary collection and analysis system offers several advantages compared to paper-based systems. The following are some of the most notable advantages.

1) Reduction of repeated data entry

During our field study, one of the most notable opportunities for time savings was during data entry. Existing data collection processes generally start with a field examiner recording data onto a paper form. This information is taken back to the lab where the data is reentered into a backend LIMS database. Also, each lab examiner writes down the information pertaining to each analysis before reentering this data into the LIMS. By enabling tablet-based electronic entry, the system would save time by allowing the examiner to enter the data once and then modify this data as needed along the way (see Figure 1).

2) Reduction in Data Errors

Another problem found during the field analysis involved data entry errors. Because users were not bound by what he or she could or could not enter, many data entry errors and inconsistencies occurred both on paper as well as during subsequent entry into the LIMS. The electronic tablet-based system helps alleviate some of these errors in two ways. First, the number of places for data entry error is kept to a minimum as reentry of data is reduced or eliminated (see Figure 1). Second, by using specific fields for certain data (calendar entry mechanism for date/time, checkbox for yes/no or true/false, etc.) the forms-based program can enforce standardization for the data entered.

3) Similarity with traditional notebook systems The tablet-based forms are designed to mimic what is already in place for forensic examination. Also, ergonomic properties of an electronic tablet will resemble traditional paper and clipboard systems. While an electronic tablet is not identical to a paper-based clipboard, the similarity will help to ease the transition to the new system as it more closely imitates extant technology.

4) Better tracking of the chain of custody for evidence items The use of a completely electronic evidence-based analysis mechanism can help to better keep track of the chain of custody. This is highly dependent on evidentiary checkout policies and procedures in each lab, but the electronic system can help to greatly augment this by keeping track of all items electronically. For example, mobile computing allows for much easier implementation of better tracking mechanisms such as barcodes and RFID technologies (see below). Second, by assigning 
specific hardware to specific individuals, data entry pertaining to evidence analysis can be cataloged as being recorded by a specific individual on a specific machine. Mechanisms now exist for identifying data as coming from a certain person and a specific hardware device using encryption and certificate technology (Luse, Townsend et al. 2008). This supplements the electronic tracking mechanisms of the physical evidence items described below with a more robust mechanism to track who has actually entered data pertaining to the analysis of an evidence item.

5) Enforces both scientific and administrative policy and procedure Because the form can be designed to only allow appropriate information and can also have the data entry sequence-controlled, it is possible to design the electronic form in such a way as to reinforce appropriate lab practices. Users attempting to enter information that (from a procedural standpoint) should follow information that is not yet entered can be prompted to complete the earlier fields. While "jumping around" on the form may just be an indicator of lab personnel entering data as they think of it, it can also be an indicator that a step may have been skipped or that necessary information has not been properly developed. Forms based computing allows managers to promulgate lab procedures quickly and in a standardized fashion, which facilitates compliance with laboratory quality control and industry standards.

6) Augments the use of $1 D$ and $2 D$ barcodes as well as RFID for evidentiary tracking

Electronic tracking of evidence items is becoming more and more common. By attaching a barcode or RFID tag to an item, it is easier to keep an "electronic eye" on each item. The prototype described in this paper was also designed to use both 2D barcodes and RFID tags for evidence analysis. Using these in tandem with forms-based computing provided a much more flexible set of tools that could be used for evidence tracking.

\subsection{System Evaluation}

Our system evaluation followed a two part process: first, we had information systems specialists that are unassociated with crime labs review the system for its technical functionality; secondly, we had crime lab personnel examine the system's operation in order to comment on it's expected utility in a forensic environment. From a technical perspective, while the system is quite basic in appearance, our reviewers found few problems with the functional operation of the system. These problems were then rectified, and the system was demonstrated to a variety of potential users in crime labs. Potential users indicated that they saw real promise in the system, both in terms of data accuracy and in terms of 
collection efficiency. The only remaining question about the usability of the system had to do with allying the forms based collection system with current information systems at their respective labs.

\subsection{Recommendations}

Due to financial, personnel, and technical constraints, many crime labs will likely encounter resistance and hurdles if the entire system were implemented at once. The following is a list of recommendations for small steps a lab can take to move towards the adoption of forms-based computing.

1) Decide upon necessary data

Many LIMS systems have many extra data fields which are never utilized. The first step is to decide which data is utilized in the current lab setting. Also, future use should be examined as you drive towards electronic notebook use. For example, are records currently kept in the LIMS regarding evidence checkout? If not, this can be implemented using an electronic system. If a new backend system will be utilized, this should be designed and implemented.

2) Make sure your LIMS system is able to use data in real time Real-time entry of data is a significant benefit in a forms-based computing system. The system should be able to handle data entry from various parties, anytime, from any location. This can be used for web-based entry (described below) and subsequent electronic tablet entry. This will provide both time savings and convenience to the lab (Verost 2007), in that there is no lag between paper entry and entry into the electronic system.

3) Implement wireless access

A first step to ease of input requires input mechanisms from any spot in the laboratory. This can be utilized by lab technicians using laptop technology and eventually electronic notebooks. The main concern here is mobility, and the outline above provides a review of the security and implementation issues involved in a wireless installation.

4) Design and implement electronic input mechanisms

Other electronic input mechanisms can provide an interim method for data entry before implementation of an electronic tablet system. The first step is to design the forms which will be used for entry. Entry methods such as web-based forms or electronic PDF forms can then be designed and implemented, and these can provide a basis for more mobile applications in the future.

5) Drive a push to anywhere entry of data

"Anywhere" entry of data deals with being able to enter data from 
anyplace at anytime. This will allow employees as well as those not directly associated with the crime lab (police officers, etc.) to enter data into the system. Once these mechanisms have been designed, you must educate and disseminate this knowledge to those who should utilize it.

6) Forms-based computing

The final step will be a forms-based computing implementation. If the above steps have been followed, then electronic forms should already be designed and will only need to be implemented for an electronic tablet. The backend system and wireless access will allow for easy integration with the current system.

\section{CONCLUSION}

The primary objective for any forensic crime lab is to process evidence as thoroughly as possible in the shortest amount of time. This has become very challenging in recent years as more in-depth analysis is possible, but which takes a greater amount of time. Also, the mandates for thorough details regarding analysis and chain of custody provide many different places for errors in record keeping. For this reason, crime laboratories need to identify where and how they can improve the data collection and management process.

In this paper, we present a method for forensic examination and analysis using an electronic tablet-based data entry system. Using a field study and subsequent prototype system development, a framework has been developed to assist those labs who are interested in implementing such a system. This includes a step-bystep framework for implementation as well as recommendations for technology use and implementation.

The electronic tablet-based system offers several advantages compared to traditional paper-based forensic analysis. Primary improvements include time savings by requiring less repeated data entry as well as data entry error improvements. Properly designed, electronic forms also enhance lab practices by ensuring that procedural steps are entered in appropriate sequence.

\section{ACKNOWLEDGMENTS}

This study and system development was made possible by Ames Lab and the Midwest Forensics Resources Center, through money grants from the National Institute of Justice. Work at the Ames Laboratory was supported by the Department of Energy under contract No. DE-AC02-07CH11358. 


\section{REFERENCES}

Ammenwerth, E., A. Buchauer, et al. (2000). "Mobile information and communication tools in the hospital." International Journal of Medical Informatics 57: 21-40.

Arnstein, L., C. Y. Hung, et al. (2002). "Labscape: a smart environment for the cell biology laboratory." IEEE Pervasive Computing 1(3): 13-21.

Faas, P., J. Seyba, et al. (2006). Collaborative logistics on the military flightline. Proceedings of the International Symposium on Collaborative Technologies and Systems, IEEE Computer Society.

Frolik, J. and J. B. Zurn (2005). "Evaluation of Tablet PCs for engineering content development and instruction." Computers in Education Journal 15(3).

Hough, D. (1989). "The paperless office (distributed processing)." Byte Magazine 14(7): 157-ff.

Hughes, G., H. Mills, et al. (2004). "The semantic smart laboratory: a system for supporting the chemical eScientist." Organic \& Biomolecular Chemistry 2(22): 3284-3293.

Hunton, J. E. (1994). "Setting up a paperless office." Journal of Accountancy 178(5): 77-85.

Lau, T. T. (1990). Building a paperless office with document image processing system. International Professional Communication Conference, 1990. IPCC 90. 'Communication Across the Sea: North American and European Practices', Guildford, UK, IEEE.

Lu, Y.-C., Y. Xiao, et al. (2005). "A review and a framework of handheld computer adoption in healthcare." International Journal of Medical Informatics 74: 409-422.

Luse, A., A. M. Townsend, et al. (2008). "Software Implementation using Hardware-Based Verification for Secure Content Delivery." Journal of Information Warfare 7(2): 11-23.

Mackay, W. E., G. Pothier, et al. (2002). The missing link: augmenting biology laboratory notebooks. Proceedings of the 15th annual ACM symposium on User interface software and technology, Paris, France, ACM.

Miller, R. H. and I. Sim (2004). "Physicians' Use Of Electronic Medical Records: Barriers And Solutions." Health Affairs 23(2): 116-126.

Myers, J. D. (2003). Collaborative Electronic Notebooks as Electronic Records: Design Issues for the Secure Electronic Laboratory Notebook (ELN). 2003 Western MultiConference, Orlando, FL, Society for Modeling and Simulation International.

Myers, J. D., C. Fox-Dobbs, et al. (1996). Electronic laboratory notebooks for 
collaborative research. 5th International Workshops on Enabling Technologies: Infrastructure for Collaborative Enterprises (WET ICE'96), Stanford, CA, IEEE.

Patterson, D. and G. Olah (1981). Using new technology for systems documentation: Specifications for an on-line documentation system for the paperless office. Proceedings of the ACM '81 conference 1981, ACM.

Phelan, S. E. (2003). "A paperless success story: a firm's use of IT to organize the office led to a digital makeover and new business." Journal of Accountancy 196(4): 59-68.

Raghupathi, W. and J. Tan (2002). "Strategic IT applications in health care." Communications of the ACM 45(12): 56-61.

Schraefel, M. C., G. V. Hughes, et al. (2004). Breaking the book: translating the chemistry lab book into a pervasive computing lab environment. Proceedings of the SIGCHI conference on Human factors in computing systems 2004, Vienna, Austria, ACM.

Sellen, A. J. and R. H. R. Harper (2002). The myth of the paperless office. Cambridge, MIT Press.

Verost, W. (2007). "Web-based LIMS: Right for any sized lab." Lab Manager 2(6): 13-16.

Walrath, J. D. (2005). Information technology for the soldier: The human factor. Army, Army Research Laboratory. Adelphi, MD.

Warner, S. (2007). "Going paperless." Lab Manager 2(6): 21-23.

Willis, C. L. and S. L. Miertschin (2004). Tablet PC's as instructional tools or the pen is mightier than the 'board! Proceedings of the 5th conference on Information technology education Salt Lake City, UT, ACM.

Wise, J. C., R. Toto, et al. (2006). Introducing Tablet PCs: Initial Results From the Classroom. 36th ASEE/IEEE Frontiers in Education Conference. San Diego, CA, IEEE: 17-20.

Wu, R. C. and S. E. Straus (2006). "Evidence for handheld electronic medical records in improving care: a systematic review." BMC Medical Informatics and Decision Making 6(26). 